\title{
The 2016 Revision of ISO 1 - Standard Reference Temperature for the Specification of Geometrical and Dimensional Properties
}

\author{
S. D. Phillips ${ }^{1}$, C. M. Shakarji ${ }^{1}$, A. Balsamo ${ }^{2}$, M. Krystek ${ }^{3}$, and E. Morse ${ }^{4}$ \\ ${ }^{1}$ National Institute of Standards and Technology, \\ Gaithersburg, MD 20899, USA \\ ${ }^{2}$ Istituto Nazionale di Ricerca Metrologica, \\ Torino, Italy \\ ${ }^{3}$ Physikalisch-Technische Bundesanstalt, \\ Braunschweig and Berlin, Germany \\ ${ }^{4}$ University of North Carolina at Charlotte, \\ Charlotte, NC 28223, USA \\ steven.phillips@nist.gov \\ craig.shakarji@nist.gov \\ a.balsamo@inrim.it \\ michael.krystek@gmx.de \\ edward.morse@uncc.edu
}

This paper discusses the changes in the 2016 (third edition) of International Standard ISO 1. While the value of the standard reference temperature remains unchanged at $20^{\circ} \mathrm{C}$, the important definitions for the "reference temperature" and "standard reference temperature," absent in prior editions, are now defined, with the latter exclusively reserved for the assignment of the internationally agreed upon temperature of $20^{\circ} \mathrm{C}$. The scope of the revised Standard has been carefully refined and made more explicit. This, together with other clarifications and improvements, has eliminated the ambiguities associated with specifications at non-standard reference temperatures and allows, if needed, different reference temperatures to be associated with different properties of a workpiece. The relationship between ISO 1 and dimensional measurements is also discussed and clarified. In this paper, we discuss the motivation for these changes and present several issues debated during the revision process for the benefit of future standards committees that might study this topic.

Key words: CTE; dimensional metrology; ISO 1; standard reference temperature, thermal expansion.

Accepted: October 3, 2016

Published: November 10, 2016

https://doi.org/10.6028/jres.121.026

\section{Introduction}

Thermal effects have long vexed dimensional metrology due to the nonzero thermal expansion of almost all materials. Changes in temperature can cause an object to expand or contract and to possibly distort, especially if the temperature distribution is non-uniform. In order to precisely specify the geometric and dimensional properties of material objects both the values of the specifications, e.g., dimensions, and their associated temperature(s) are required. Further, to avoid confusion and to facilitate design, production, 
and commerce, a fixed internationally accepted standard reference temperature applicable for all geometric and dimensional properties is specified for material objects by the International Standard ISO 1 [1].

The standard reference temperature for length measurements has a long history. The Platinum-Iridium Meter of the Archives, which was the standard of length between 1889 and 1960, had the meter defined as the distance between two scribed lines while at the temperature of the melting point of ice. While this temperature provided a well-defined and reproducible length standard, it was very inconvenient for performing dimensional metrology. As engineering tolerances on mechanical components tightened, the goal of an international reference temperature near room temperature became increasingly critical. Doiron [2] reviews the early $20^{\text {th }}$ century efforts to develop a consensus for a "near room temperature" standard reference temperature for length and notes that at the 1931 meeting of the International Committee for Weights and Measures (CIPM), the Committee adopted a $20^{\circ} \mathrm{C}$ reference temperature for industrial length measurements. In 1954, the ISO published ISO Recommendation ${ }^{1} \mathrm{R} 1$ [3], which was adopted in 1975 as International Standard ISO 1 [4], stating "The standard reference temperature for industrial length measurements is fixed at $20^{\circ} \mathrm{C}$." A footnote explains that "a metre gauge with the indication: reference temperature $20{ }^{\circ} \mathrm{C}$ has at $20^{\circ} \mathrm{C}$ the same length as the international prototype at $0{ }^{\circ} \mathrm{C}$." The footnote was reproduced in 1975 from the 1954 R1 recommendation despite that the International prototype had been retired by 1960 and the meter was then defined by the wavelength of light.

The 2002 edition of ISO 1 [5] states that "The standard reference temperature for geometrical product specification and verification is fixed at $20^{\circ} \mathrm{C}$." The expansion of the scope to "geometrical product specification and verification" was intended to clarify some issues, for example, that all geometrical and dimensional specifications were subject to the $20^{\circ} \mathrm{C}$ reference temperature, not just length measurements. (Deductive logic applied to the 1975 ISO 1 standard would conclude that if length was subject to a $20^{\circ} \mathrm{C}$ standard reference temperature then all geometric and dimensional specifications, such as form, angle, and surface texture, were also subject to the same standard reference temperature; nevertheless, this was often a question in the mind of industrial practitioners.) Unfortunately, the enlarged scope created additional confusion, such as a debate whether indicating dimensional measuring instruments should have their performance specifications, i.e., their accuracy, specified only at $20^{\circ} \mathrm{C}$, as was customary for material standards, such as gage blocks. This, and other issues described below, motivated ISO Technical Committee (TC) 213 on "dimensional and geometrical product specifications and verification" to instruct Working Group (WG) 4 on "uncertainty of measurement and decision rules" to initiate Ref. [6] work (per the resolution in Ref. [6]) to revise ISO 1. The objectives of the revision include: (1) to make explicit that ISO 1 applies to all geometric and dimensional properties, e.g., flatness, and not just length; (2) to create distinct and separate terminology for a "reference temperature" and the "standard reference temperature" with the latter exclusively used for the internationally fixed temperature of $20^{\circ} \mathrm{C}$; (3) to clarify the scope of ISO 1 whereas earlier editions implied that ISO 1 applied to all industrial length measurements (not always true, as discussed in Sec. 3 ) and the prior edition implied that ISO 1 applied to all parameters of ISO TC213 Standards (not always true, as discussed in Sec. 3); and (4) to clarify that the role of ISO 1 in geometric and dimensional measurements is through the definition of the measurand of the measurement. The authors were part of the project team that drafted the 2016 revision of ISO 1 [1]. This paper discusses the changes to ISO 1, provides motivation for these changes, and records several of the debated issues for the benefit of future standard committees that might study this topic.

\section{Standard Reference Temperature}

There is no change in the value of the standard reference temperature-it remains fixed at $20^{\circ} \mathrm{C}$. However, the concept of the reference temperature is defined and broadened relative to prior editions. The International vocabulary of basic and general terms in metrology (VIM) [7] makes a careful distinction between a quantity (VIM 1.1), i.e., a property of a phenomenon, body, or substance, and its quantity value (VIM 1.19), i.e., the value of the quantity. In previous editions of ISO 1, the standard reference temperature was not defined and only assigned a quantity value of $20^{\circ} \mathrm{C}$. Unfortunately, this provided neither a clear

\footnotetext{
${ }^{1}$ Prior to 1972 the work of ISO technical committees was published as ISO recommendations.
} 
definition of the meaning of the standard reference temperature nor any unambiguous means to employ a different reference temperature. The 2016 ISO 1 edition separates this issue into three components: a definition for the reference temperature, a definition for the standard reference temperature, and an assignment of a value to the standard reference temperature.

\section{Issues of Scope}

The scope of the ISO R1-1954 and ISO 1-1975 editions applying to "industrial length measurements" had always been incorrect in certian applications. Many industrial length measurements do not apply the standard reference temperature, but instead determine the distance between two points at the moment the measurement is taken. This uses the definition of the meter, which is temperature independent, and the measurand implicitly refers to the conditions that exist at the moment of measurement. For example, optically based length measurements using interferometers, laser trackers, or laser scanners, measure the distance from the instrument to a target, and this does not require the use of the standard reference temperature, and any measurement of the ambient temperature is used only to compensate for the index of refraction of the air and has nothing to do with applying the standard reference temperature. Many civil engineering measurements, e.g., surveying — whether by GPS, time-of-flight, or measuring tape — do not apply the standard reference temperature to the object they are measuring, i.e., the earth. (In the case of measuring tapes, the tape is calibrated at the standard reference temperature and in high accuracy applications it is corrected for its temperature back to $20^{\circ} \mathrm{C}$, but the measurand-the distance between two points on the earth—does not involve the standard reference temperature.) Indeed, just performing a measurement to determine if a box will fit through a doorway is a measurement where the measurands are defined as the distances at the moment of measurement and do not involve the standard reference temperature. In fact, it could be that a box will fit through a doorway at elevated temperatures even though the corresponding dimensions at $20^{\circ} \mathrm{C}$ would indicate the opposite.

When revising the scope of ISO 1 , WG 4 declined suggestions that $20^{\circ} \mathrm{C}$ be the default reference temperature for all dimensional measurements, based on the reasons described above for avoiding the "[all] industrial length measurements" scope of earlier editions. However, the body of the 2016 version of the Standard does state that "unless otherwise explicitly specified the reference temperature for geometrical and dimensional properties of workpieces shall be the standard reference temperature" [1] where the default is specifically restricted to the case of workpieces and the emphasis is on the specification of geometrical and dimensional properties.

Among the most important applications of the standard reference temperature is the specification of dimensional and geometric properties of workpieces such as size, orientation, location, and form (i.e., shape). Billions of mechanical workpieces are annually produced based on the meaning of the numbers shown in their specifications, as defined by mechanical drawings (or more recently on computer-based solid models) and these numbers have a well-defined meaning only when associated with a reference temperature. In fact, a strong argument was made that ISO 1 only applies to the specification process and that any subsequent dimensional measurements, i.e., verifications, are just following the instructions given in the specifications. Even in the case of measuring an object that has no associated dimensional specification (e.g., no drawing), it was argued that a meaningful measurement must have a well-defined measurand and defining the measurand creates a dimensional specification, where the measurement results become the values, e.g., dimensions, of the specification and they have an associated reference temperature. That is, the measurand combined with the measurement results could be displayed in the form of a mechanical drawing specification with the expanded measurement uncertainties as the tolerances. This argument — that the text of ISO 1 should be limited solely to dimensional specifications — while logically valid, was ultimately considered too arcane to include in the Standard for most industrial users who usually think in terms of measurement results.

The 2002 edition of ISO 1 tried to address the scope issue by expanding it to "geometrical product specification and verification." Unfortunately, this is the name of TC 213 and all Standards from this TC have titles that begin with "geometrical product specifications." Standards from TC 213 cover many applications and can specify a wide range of properties, including non-dimensional properties such as the 
hardness of surfaces, or even electrical or optical properties; clearly not all of these involve the $20{ }^{\circ} \mathrm{C}$ reference temperature and some of these quantities are defined by a different reference temperature. More importantly, TC 213 produces many Standards that specify the accuracy of dimensional measuring instruments. Arguments were raised that the accuracy specifications given by TC 213 Standards should only be stated at the standard reference temperature according to ISO 1. However, industrial users of dimensional measuring instruments are frequently interested in the instrument's accuracy at the ambient temperatures likely to be present in their measurement applications. The measurand associated with an instrument's accuracy specification is the measurement error made by the instrument when measuring a specified reference quantity within the instrument's rated operating conditions and, in particular, within the rated ambient temperature range. The measurement error is defined as the instrument's indicated value (at the moment and temperature of the measurement) minus the (true) value of the dimensional reference quantity, where the value of the dimensional reference quantity is given at the standard reference temperature. So the connection to ISO 1 is through the definition of the reference quantity, i.e., the reference quantity has its geometrical and dimensional property values defined at $20^{\circ} \mathrm{C}$. This does not require that the instrument's measurement error be defined only at $20^{\circ} \mathrm{C}$; the instrument's measurement error is well-defined over its entire rated operating conditions.

The scope of the 2016 ISO 1 edition "defines the concepts of a reference temperature and of the standard reference temperature, and specifies the standard reference temperature value for the specification of geometrical and dimensional properties of an object.” These definitions (discussed later) can be employed by any other ISO Standards or dimensional specifications through citation. The scope was also explicitly made "applicable to the definition of the measurand used in verification or calibration" in order to address the common understanding of applying ISO 1 to measurement results. The sentence carefully states that the relationship with ISO 1 is through the definition of the measurand and not through the measurement process. In particular, this means that neither the object under measurement nor the measuring instrument must be at $20^{\circ} \mathrm{C}$ during the measurement; however, the consequences of not measuring at the standard reference temperature must be included in the uncertainty evaluation associated with the measurement result.

Finally, the 2016 scope is specifically restricted to the application of dimensional and geometric properties of an object and avoided the open-ended terms such as "industrial measurements" or "product specifications." Examples of dimensional and geometric properties of an object are given in the scope and include size, location, orientation (including angle), form, and surface texture of a workpiece. Instrument performance specifications are specifically not included, as they are generally not dimensional or geometric properties of an object.

\section{Fundamental Definitions}

One of the objectives of the current revision is to provide definitions for a "reference temperature" and also for the "standard reference temperature." The prior editions omitted the definitions and only provided an assigned value of $20^{\circ} \mathrm{C}$ to the standard reference temperature, which is equivalent to the algebraic statement that $x=4$; which tells us the value of $x$ but nothing about what $x$ represents. In particular, it was unclear if the standard reference temperature was just a default that could be changed as needed, or if it was an immutable, internationally assigned value. This ambiguity leads to difficulties when trying to communicate a non-standard reference temperature. There are good design reasons (discussed in Sec. 5) why, in certain cases, it is useful to have a reference temperature different from the standard reference temperature. Consequently, ISO 1: 2016 defines a "reference temperature" (within the scope of dimensional and geometric properties of an object) as "the temperature of an object, having a uniform temperature, specified in the definition of a geometrical or dimensional property." The Standard then defines the "standard reference temperature" as an "internationally agreed-upon reference temperature." Therefore, in special situations, it is possible to unambiguously specify workpieces with geometric and dimensional characteristics defined at a reference temperature other than the standard reference temperature, or even to have each geometric or dimensional property of a workpiece specified at a different reference temperature if needed. This flexibility is important, even though for most cases, all properties of 
the object will be defined with the standard reference temperature, which is now clearly unique and internationally specified.

\section{Applications Using Different Reference Temperatures}

In special situations, the use of the standard reference temperature can result in a suboptimal functional design. Consider a precision mechanical component that functions in a constant temperature environment that is not at the standard reference temperature, e.g., a heart pump that functions at the human body temperature of $37^{\circ} \mathrm{C}$. If the geometric and dimensional properties of this component are specified at the standard reference temperature, then the designer of this component must estimate what the actual geometric and dimensional properties of the component will be at its functional temperature. This involves integrating the coefficient of thermal expansion (CTE) function from the standard reference temperature to the functional temperature, and the CTE is often poorly known for many materials [8]. Even for gage block steel, a well characterized material in dimensional metrology applications, the CTE value is typically known only within $\pm 1 \times 10^{-6} /{ }^{\circ} \mathrm{C}$ [9]. The result is a design that has uncertain geometric and dimensional properties at the functional temperature that could result in a suboptimal component. A simple solution to this problem is to specify a reference temperature that is equal to the functional temperature of the component. The design is now uniquely specified and provides the unambiguous geometric and dimensional properties required for function. The complications of a precision component with a reference temperature specified different from the standard reference temperature are transferred to the manufacturing and metrology organizations, but they are in a better position than the designer to address this issue. For example, the CTE of a metal often varies significantly between different production runs of the same metal, but within a single run the CTE is more uniform. Therefore, by measuring the CTE of a few metal samples the uncertainty in the CTE for the particular metal batch used in manufacturing the component can be greatly reduced. Machining operations (and subsequent measurements) can use the measured CTE value in their thermal compensation calculations to improve the accuracy of their results. Another option would be to increase the temperature of manufacturing and measurement operations to the specified reference temperature and achieve the required accuracy directly. In either case, when trying to achieve the highest possible functionality, the issue is often better addressed by the manufacturing and measurement departments rather than by the designer-who must either estimate the consequences of the thermal expansion or impose very expensive requirements on the CTE value of the component material.

Different technical disciplines often have their own standard reference conditions. In some cases, there may be overlap between the application of different Standards and this can create confusion unless the specifications are unambiguously communicated. For example, chemists often designate $0{ }^{\circ} \mathrm{C}$ as the standard temperature for defining volumes of gasses [10]; commercial transactions of natural gas are typically measured in units of cubic meters at a standard temperature of $15^{\circ} \mathrm{C}$ [11]; and the "standard cubic foot" of gasses (other than petroleum gasses) often has a reference temperature of $21^{\circ} \mathrm{C}$ [12]. The manufacturing of a physical embodiment of a unit of volume to contain a gas would need to be carefully specified to avoid misunderstandings. Because the scope of ISO 1 specifies the standard reference temperature value of $20^{\circ} \mathrm{C}$ for all geometrical and dimensional properties of an object, all the respective geometrical and dimensional specifications (e.g., mechanical drawings) of the physical container are subject to ISO 1 unless otherwise explicitly specified. If the designer needed an exact specification at a nonstandard reference temperature (and thus avoiding the thermal expansion uncertainty as described above), it can now be unambiguously specified; in no case should the phase "standard reference temperature" be used for any meaning other than the internationally agreed upon value of $20^{\circ} \mathrm{C}$ when specifying the geometrical and dimensional properties of an object.

In some unusual cases, multiple reference temperatures may be needed on a component. For example, high-frequency microwave waveguides are metallic tubes with precise dimensions. Electrical calibration laboratories operate at $23^{\circ} \mathrm{C}$ (the reference temperature for electrical quantities) and to achieve an exact specification for the internal waveguide dimensions at its operating temperature, a reference temperature of $23^{\circ} \mathrm{C}$ is required. Other geometrical features on the waveguide, e.g., external dimensions, could be specified at the standard reference temperature of $20^{\circ} \mathrm{C}$. This bifurcation allows the additional production 
and measurement effort to be focused on just the internal waveguide geometric and dimensional characteristics. Another example would be a specification of the flatness of a bimetal plate where opposite sides of the plate are made of materials with different CTEs. A flat plate may be functionally required at a non-standard reference temperature while its size might be specified at the standard reference temperature.

\section{Summary}

The 2016 revision of ISO 1 now specifies the meaning of the reference temperature and the standard reference temperature used to specify geometric and dimensional properties of an object and maintains the long established value of the standard reference temperature at $20^{\circ} \mathrm{C}$. The new definitions also allow an unambiguous specification of a reference temperature that is different from the standard reference temperature, and even facilitates the specifications of different reference temperatures for different geometric and dimensional properties of an object.

\section{Acknowledgments}

The authors would like to thank all the members of ISO TC213/WG4 for their efforts, especially Dr. Henrik Nielsen (chair of ISO/TC 213/WG 4) for his guidance on this Standard and Mr. Peter Damgaard (secretary of ISO/TC 213/WG 4) for his expeditious assistance; we also thank Mr. Thomas Charlton (chairman of the ASME B89 committee on dimensional metrology) for his thoughtful discussions.

\section{References}

[1] International Organization for Standardization (2016) ISO 1:2016 - Geometrical product specifications (GPS) - Standard reference temperature for the specification of geometrical and dimensional properties (International Organization for Standardization, Geneva, Switzerland). Available https://www.iso.org/obp/ui/\#iso:std:iso:1:ed-3:v1:en. Accessed November 3, 2016.

[2] Doiron T (2007) 20 degrees C - A short history of the standard reference temperature for industrial dimensional measurements. J Res Natl Inst Stan 112(1):1-23. https://doi.org/10.6028/jres.112.001

[3] International Organization for Standardization (1954) ISO Recommendation R1 - Standard reference temperature for industrial length measurements (International Organization for Standardization, Geneva, Switzerland), $1^{\text {st }}$ Ed.

[4] International Organization for Standardization (1975) ISO 1:1975 - Standard reference temperature for industrial length measurements (International Organization for Standardization, Geneva, Switzerland), $1^{\text {st }}$ Ed.

[5] International Organization for Standardization (2002) ISO 1:2002 - Geometrical Product Specifications (GPS) - Standard reference temperature for geometrical product specification and verification (International Organization for Standardization, Geneva, Switzerland), $1^{\text {st }}$ Ed.

[6] International Organization for Standardization Technical Committee 213 (2013) Resolutions Adopted at the 34 ${ }^{\text {th }}$ meeting of ISO/TC 213, 2013-02-22 - Berlin, Germany (International Organization for Standardization, Geneva, Switzerland). Available http://isotc213.ds.dk/agensum/ISOTC213N1582_Resolutions_Berlin_2013.pdf. Accessed November 3, 2016

[7] Bureau International des Poids et Mesures-Joint Committee for Guides in Metrology (2012) International Vocabulary of Metrology — Basic and General Concepts and Associated Terms (VIM). JCGM 200:2012. Available www.bipm.org/en/publications/guides/vim.html. Accessed November 3, 2016.

[8] Swyt DA (1994) Uncertainties in Dimensional Measurements Made at Nonstandard Temperatures. J Res Natl Inst Stan 99(1):31-44. https://doi.org/10.6028/jres.099.004

[9] The American Society of Mechanical Engineers (2002) B89.1.9-2002 - Gage Blocks, Sec. 6.2.2 (The American Society of Mechanical Engineers, New York, NY). Available https://www.asme.org/products/codes-standards/b8919-2002-gage-blocks. Accessed November 3, 2016.

[10] McNaught AD, Wilkinson A, International Union of Pure and Applied Chemistry. (1997) Compendium of chemical terminology: IUPAC recommendations (Blackwell Science, Oxford, England; Malden, MA), $2^{\text {nd }}$ Ed. XML on-line corrected version (2006). Available at http://goldbook.iupac.org. Accessed November 3, 2016.

[11] International Organization for Standardization (1996) ISO 13443:1996 Natural gas - Standard reference conditions (International Organization for Standardization, Geneva, Switzerland). Available https://www.iso.org/obp/ui/\#!iso:std:20461:en. Accessed November 3, 2016.

[12] National Institute of Standards and Technology Office of Weights and Measures (2016) Uniform Laws and Regulations in the Areas of Legal Metrology and Engine Fuel Quality (U.S. Department of Commerce, Washington, D.C.), NIST Handbook 130, Section 2.16. https://doi.org/10.6028/NIST.HB.130-2016 
About the authors: Steve Phillips is a physicist in the Dimensional Metrology Group at the National Institute of Standards and Technology and is the U.S. primary subject matter expert for ISO TC213/WG4 who lead the revision of ISO 1. Craig Shakarji is an applied mathematician in the Dimensional Metrology Group at the National Institute of Standards and Technology and is a subject matter expert for ISO TC 213/WG4. Sandro Balsamo is a senior dimensional metrologist at the (Italian) National Institute for Research in Metrology, INRIM, and is the Italian primary subject matter expert for ISO/TC213/WG4. Michael Krystek is a recently retired physicist from the German national metrology institute PTB, he is still involved in standardization work. Edward Morse holds the rank of professor in mechanical engineering at UNC Charlotte and is the deputy director of UNC Charlotte's Center for Precision Metrology; Professor Morse is a subject matter expert in ISO TC213 WG 4. The National Institute of Standards and Technology is an agency of the U.S. Department of Commerce. 